\title{
Investigation the natural convection heat transfer between two fluids inside square enclosure separated by thin plate
}

\author{
Dr. Amir S. Dawood \\ Assistant professor \\ Raed Ahmed Ali \\ Lecturer \\ Ramiz Ibraheem Saeed \\ University of Mosul- Engineering College -Mechanical Engineering Department
}

\begin{abstract}
Fluid flow and heat transfer in a square enclosure diagonally divided by a conductive thin plate has been numerically investigated. The upper diagonal zone of the enclosure filled with a saturated porous medium while the subdiagonal zone of the enclosure filled with air as working fluids. The enclosure was heated partially from below and cold from upper surface isothermally while the two vertical surface are insulated. The governing equations are solved using finite difference methods. Results were obtained for different value of Rayleigh number $\left(10^{3} \leq \mathrm{Ra} \leq 10^{5}\right)$ for air and different value of modified Rayleigh number $\left(100 \leq \mathrm{Ra}^{*} \leq 500\right)$ for porous medium as well as different dimensionless length of the heated surface $(0.2,0.4,0.5$ and 0.6$)$. The results show that the increasing in Rayleigh number in both sides of the enclosure (Ra* $\&$ Ra) causes increasing in the Nusselt number. The percentage increasing is approximately is $\left(92 \%\right.$ at $\mathrm{Ra}^{*}=100,1000 \leq \mathrm{Ra} \leq 10^{5}$ and $71 \%$ at $\mathrm{Ra}^{*}=500,1000 \leq \mathrm{Ra} \leq 10^{5}$ for $\mathrm{S}=1)$ and $\left(90 \%\right.$ at $\mathrm{Ra}^{*}=100, \mathrm{Ra}=10^{4}$ and $0.2 \leq \mathrm{S} \leq 0.6$ and $80 \%$ at $\mathrm{Ra}{ }^{*}=500, \mathrm{Ra}=10^{4}$ for $0.2 \leq \mathrm{S} \leq 0.6$ ). The flow become multi-cellular in porous media side also the increasing in dimensionless length of the heated surface causes increasing in the Nusselt number.

Keywords: natural convection; enclosure; thin plate; two fluids
\end{abstract}

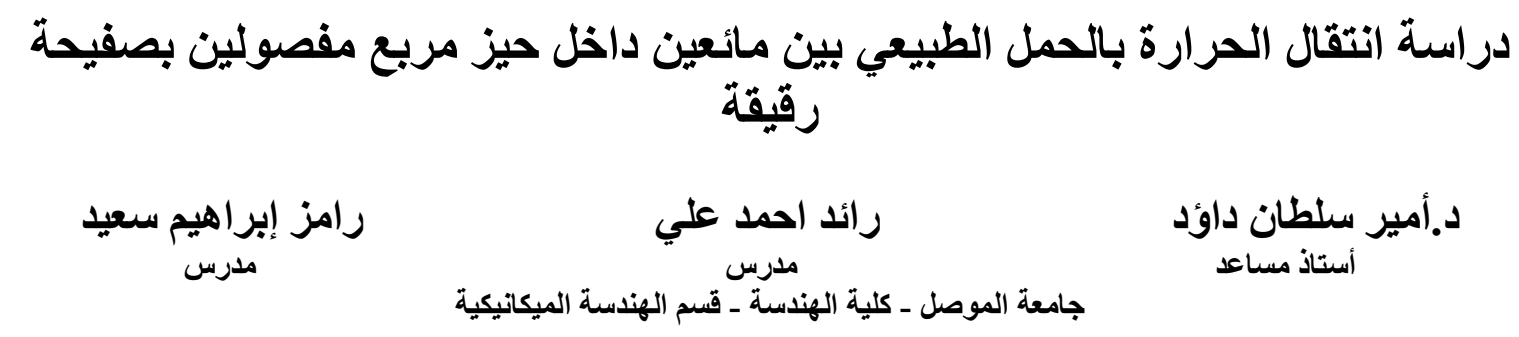

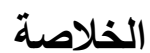

دراسة عداية لانسياب المائع وانتقال الحرارة بالحمل الطبيعي داخل حيز مربع مقسم قطريا بصفيحة رقيقة

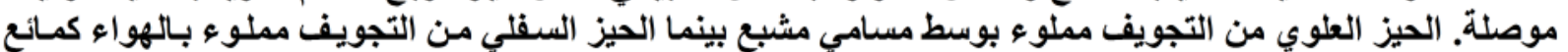

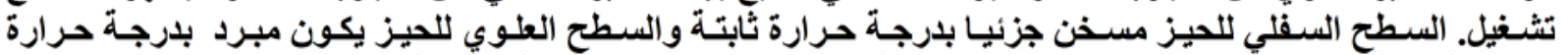

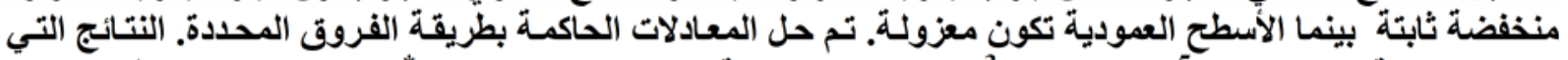

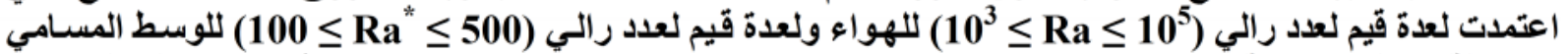

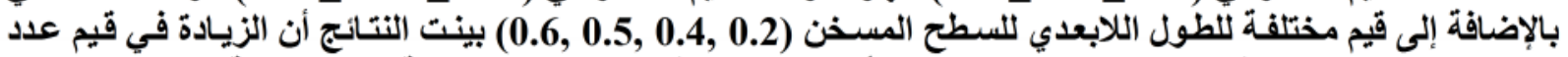
رالي (Ra* \& Ra) في كلا الحيزين من التجويف أدت إلى زيادة في عدد نسلت بنسبة زيادة مئوية ( $\left(\operatorname{Ra}^{*}=500,1000 \leq \operatorname{Ra} \leq 0^{5}\right.$ for $\left.S=1\right) \quad(71 \%) \quad\left(\operatorname{Ra}^{*}=100,1000 \leq \operatorname{Ra} \leq 0^{5}\right.$ for $\left.\left.S=1\right)\right)$

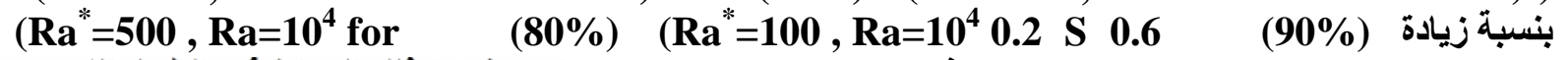
ت ت متويف وكنلك الزيادة في الطول اللابعدي

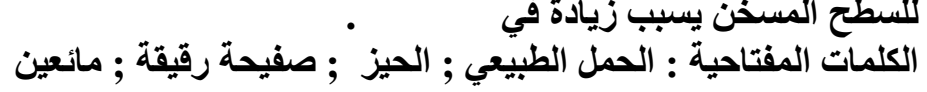




\section{Nomenclature}

g gravitational acceleration, $\mathrm{m} / \mathrm{s}^{2}$

S heated surface length

$\hat{S}$ Dimensionless heated surface length

$\mathrm{H}$ height of enclosure, $\mathrm{m}$

$\mathrm{L}$ width of the enclosure, $\mathrm{m}$

$\mathrm{Nu}$ mean Nusselt number

Pr Prandtl number

$\mathrm{Ra}$ Rayleigh number in air side

Ra* modified Rayleigh number in porous media side

$\mathrm{T}$ temperature, $\mathrm{K}$

$\mathrm{u}, \mathrm{v}$ axial and radial velocities, $\mathrm{m} / \mathrm{s}$

$\hat{x}, \hat{y} \quad$ Non-dimensional coordinates

\section{Greek symbols}

$\boldsymbol{9}$ kinematic viscosity, $\mathrm{m}^{2} / \mathrm{s}$

$\boldsymbol{\theta}$ non-dimensional temperature non-dimensional vorticity

$\boldsymbol{\beta}$ thermal expansion coefficient, $1 / \mathrm{K}$

$\boldsymbol{\alpha}$ thermal diffusivity, $\mathrm{m}^{2} / \mathrm{s}$

$\hat{\psi}$ non-dimensional stream function

$\omega$ vorticity

\section{Introduction}

A problem of considerable fundamentals and practical interest is that concerning transport phenomena resulting from natural convection in a composite system consisting of a fluid and porous medium saturated with the same fluid. Studies of these phenomena have intensified in recent year because of the wide range of application associated with naturally occurring and industrial processes such as geothermal energy systems, the cooling and fumigation of stored food grains, the cooling of aluminum billets, filtration and so on [1].

Yasin Varol [2] A numerical work was performed to determine the heat transfer and fluid flow due to buoyancy forces in divided trapezoidal enclosures filled with fluid saturated porous media. Results are presented for different values of the governing parameters, such as Rayleigh number for a porous medium, location of the partition, thickness of the partition and thermal conductivity ratio between solid and fluid media. It was observed that the conduction mode of heat transfer became dominant inside the cavity for higher thickness of the partition, low Rayleigh numbers, and low thermal conductivity ratio.

A.M. Aramyo et al [3] The flow pattern and heat transfer in a pair of cavities stacked one over the other, with a separating solid plate in between, are numerically studied. The configuration idealizes two trays in a multiple effect desalinator. The work focuses on the computational modeling of the two conjugate recirculations in the cavities, conductively coupled through the thin separating plate. The effect of the plate physical parameters, thickness and conductivity, on the heat transfer are also analyzed. Attention was paid to the temperature variation over, and heat flow through the separating plate. Global Nusselt numbers are calculated for the bottom side of the lower cavity. Data are obtained for air as the working fluid $(\operatorname{Pr}=0.7)$ in the range $10^{3}<\operatorname{Ra}<10^{7}$

Hakan F. et al [4] the flow and heat transfer due to buoyancy forces in a square enclosure divided by an impermeable partition between air and water filled chests numerically analyzed by using a finite difference technique. The enclosure was heated from left wall and cooled from right, isothermally. Horizontal walls were adiabatic. The partition divided the enclosure into air and water regions. Thus, two cases were examined: left side of partition was filled with air and right side was filled with water (Case I, air-partition- water) and left side was filled with water and right side with air (Case II, water-partition-air). Epoxy was chosen as partition material. Results were obtained for different Grashof numbers $\left(10^{3} \leq \mathrm{Gr} \leq 10^{6}\right)$, thickness of the partition $(0.05 \leq \mathrm{e} \leq 0.2)$ and location of the partition $(0.25 \leq \mathrm{c} \leq 0.75)$. An 
analytical treatment has been performed for low Grashof numbers. Numerical and analytical results gave an acceptable agreement. It was found that filling of fluid into chests is important for obtaining maximum heat transfer and energy saving. When left chest was filled with air (Case I), higher heat transfer was formed. It was an interesting result that heat transfer decreased with increasing of location of the partition for all values of partition thickness at Case I. On the contrary, heat transfer was a decreasing function of increasing value of location of the partition.

Hakan F. et al [5] the conduction-combined forced and natural convection (mixed convection) heat transfer and fluid flow have been performed for 2-D lid-driven square enclosure divided by a partition with a finite thickness and finite conductivity numerically studied. This investigation covers a wide range of Richardson number which is changed from 0.1 to 10 , thermal conductivity ratio varies from 0.001 to 10 . It is observed that higher heat transfer was formed for higher Richardson number for upward moving wall for all values of thermal conductivity ratio. When forced convection becomes effective, the orientation of moving lid becomes insignificant. Heat transfer is a decreasing function of increasing thermal conductivity ratio for all cases and Richardson numbers.

M. Mamou et al [6] Laminar natural convection heat transfer is studied in a tilted fluid system consisting of multiple layers of fluid separated by solid partitions with finite thickness and conductivity. Results are obtained in terms of overall Nusselt number $\mathrm{Nu}$ as a function of Rayleigh number Ra, angle of inclination of the system $\Phi$, solid to fluid conductivity ratio $K$, thickness of the fluid layer ' $\mathrm{Zi}$ ' thickness of the solid partitions ti and number of partitions $\mathrm{N}$. The critical Rayleigh number for the onset of convection in a bottom-heated horizontal system is predicted. The results are compared with limiting cases of the problem and are in agreement.

C. Beckermann et al [7] studied numerically and experimentally is performed to analyze the steady-state natural convection fluid flow and heat transfer in a vertical rectangular enclosure that is partially filled with a vertical layer of a fluid-saturated porous medium. It is found that the amount of fluid penetrating from the fluid region into the porous layer depends strongly on the Darcy ( $\mathrm{Da}$ ) and Rayleigh ( $\mathrm{Ra}$ ) numbers. For a relatively low product of $\mathrm{Ra}$. Da, the flow takes place primarily in the fluid layer, and heat transfer in the porous layer is by conduction only. On other hand, fluid penetrating into a relatively highly permeable porous layer has a significant impact on the natural convection flow patterns in the entire enclosure.

Nikolay P. Moshkin [8] fluid flow and heat transfer in a two-layer system of an immiscible incompressible fluid numerically studied. The results of the two dimensional numerical simulation are compared with the experimental data of the hydrodynamics and heat exchange within a horizontal two-layer medium consisting of two immiscible liquids of different densities and viscosities. Comparisons are made for the profiles of the temperature and for the profiles of the horizontal and vertical components of the velocity vector as well. Qualitative agreement between numerical and experimental results is observed.

Aniruddha Bagchi et al [9] numerical studied of two-dimensional natural convection in fluid-superposed porous layers heated locally from below is reported based on the onedomain formulation of the conservation equations. The result shows that the Nusselt numbers increase with a decrease in the heater length and height ratio, and increase with the Darcy number. The size of the heat source does not affect the dependence of the heat transfer coefficient on height ratio and Darcy number. For domains with large aspect ratios, complex flow restructuring is observed with an increase in Rayleigh number. 
M. Jafari et al [10] natural convection in a square enclosure in which two porous media are separated by a vertical solid wall This paper numerically examined. The results show that a higher heat transfer rate is achieved as the solid wall moves away from the centre of the enclosure. Moreover, at high Rayleigh numbers, the heat transfer rate increases as the thickness of the solid wall increases. It is also shown that the thermal conductivity and permeability of the solid wall affect the heat transfer mechanism within the enclosure.

Zineddine Alloui et al [11] the stability and natural convection in a system consisting of a horizontal fluid layer over a layer of saturated porous medium analytically studied. An analytical solution is obtained using a parallel flow approximation, for constant-flux thermal boundary conditions, for which the onset of supercritical cellular convection occurs at a vanishingly small wave number and can thus be predicted by the present theory. The critical Rayleigh number, Rac, and Nusselt number, $\mathrm{Nu}$, are found to depend on the depth ratio, $\eta$, the Darcy number, Da, the thermal conductivity ratio, $\gamma$ and the slip parameter $\alpha$. Results are presented for a wide range of each of the governing parameters. The results are compared with limiting cases of the problem and are found to be in agreement.

M. Bargach et al [12] Natural convection fluid flow and heat transfer in a shallow rectangular cavity containing two stratified fluid layers, one atop the other, is investigated numerically. It is demonstrated that five different patterns of convection can be observed in the present system. Velocity and temperature distributions, stream function, and local and average Nusselt numbers are presented over a wide range of governing parameters.

The main purpose of this study is to investigate the natural convection heat transfer and fluid flow inside two dimensional square enclosure fully divided diagonally with a conductive thin plate which is filled with two different fluid ( air - Porous medium ) as working fluids.

\section{Physical model}

Physical model is given in Figure (1) with coordinates and boundary conditions. In this figure, cross-section of the enclosure is a square with $\mathrm{H}=\mathrm{L}$ and is divided diagonally into two triangles by a conductive thin plate. It is heated partially from lower surface and cooled from the upper surface while left and right vertical walls are adiabatic. The left triangle enclosure is filled with air as a working fluid and the right one filled with porous medium saturated with fluid.

\section{Mathematical Formulation}

\section{1 porous medium layer}

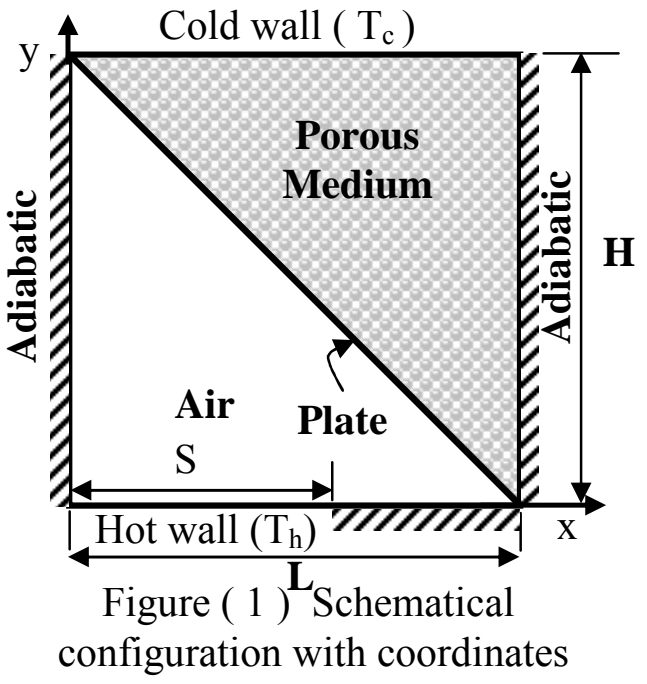

The porous media is assumed to be rigid and the fluid incompressible, all other fluid and solid properties are assumed constant, fluid and matrix are in thermal equilibrium and isotropic, the porous medium is homogeneous also the upper and lower face of the thin inclined conductive plate at the same temperature, the flow assumed two dimensional, steady state, the viscous dispersion assumed is neglected and no heat generation and the Darcy's law is assumed valid. 
The governing equations that describe the steady state heat transfer process of laminar natural convection in the porous media layer with Darcy flow and Boussinesq approximation. The governing equations are continuity, momentum and energy.

$$
\begin{aligned}
& \frac{\partial u}{\partial x}+\frac{\partial v}{\partial y}=0 \\
& \frac{\partial u}{\partial y}-\frac{\partial v}{\partial x}=-\frac{\rho_{o} g \beta K}{\mu} \frac{\partial T}{\partial x} \\
& u \frac{\partial T}{\partial x}+v \frac{\partial T}{\partial y}=\alpha_{e}\left[\frac{\partial^{2} T}{\partial x^{2}}+\frac{\partial^{2} T}{\partial y^{2}}\right]
\end{aligned}
$$

\subsection{Air layer}

the assumptions for air layer to simplify the analysis are made the fluid is incompressible, steady and laminar flow, there is no viscous dissipation, the gravity acts in the vertical direction, fluid properties are constants and fluid density variations are neglected except in the buoyancy term (the Boussinsque approximation ), and radiation heat exchanges is negligible.

The governing equations include the equations of the continuity, momentum equations and the energy equation. The equations are presented below.

1-The continuity equation:

$$
\frac{\partial u}{\partial x}+\frac{\partial v}{\partial y}=0
$$

2-The energy equation:

$$
\rho c\left(u \frac{\partial T}{\partial x}+v \frac{\partial T}{\partial y}\right)=\frac{\partial}{\partial x}\left(k \frac{\partial T}{\partial x}\right)+\frac{\partial}{\partial y}\left(k \frac{\partial T}{\partial y}\right)
$$

3-The momentum equation in $\mathrm{x}$ and $\mathrm{y}$ direction are :

$$
\begin{aligned}
& u \frac{\partial u}{\partial x}+v \frac{\partial u}{\partial y}=-\frac{1}{\rho} \frac{\partial p}{\partial x}+\frac{\partial}{\partial x}\left(\mu \frac{\partial u}{\partial x}\right)+\frac{\partial}{\partial y}\left(\mu \frac{\partial u}{\partial y}\right)-\rho g \\
& u \frac{\partial v}{\partial x}+v \frac{\partial v}{\partial y}=-\frac{1}{\rho} \frac{\partial p}{\partial y}+\frac{\partial}{\partial x}\left(\mu \frac{\partial v}{\partial x}\right)+\frac{\partial}{\partial y}\left(\mu \frac{\partial v}{\partial y}\right)
\end{aligned}
$$

Where $\mathrm{k} \& \mu$ are constants 
with Boussinesq approximations, the density is constant for all terms in the governing equations except for the bouncy force term that the density is a linear function of the temperature.

$\rho=\rho_{\circ}(1-\beta \Delta T)$

by introducing equation 8 into equation 6 yield:

$u \frac{\partial u}{\partial x}+v \frac{\partial u}{\partial y}=-\frac{1}{\rho} \frac{\partial p}{\partial x}+\frac{\partial}{\partial x}\left(\mu \frac{\partial u}{\partial x}\right)+\frac{\partial}{\partial y}\left(\mu \frac{\partial u}{\partial y}\right)-g \beta \Delta T$

\subsection{The separating plate}

The energy equation for the conductive thin plate is [ 2 ].

$\frac{\partial^{2} T}{\partial x^{2}}+\frac{\partial^{2} T}{\partial y^{2}}=0.0$

The stream function $(\psi)$ and vorticity $(\omega)$ in the governing equations are defined as follows:

$u=\frac{\partial \psi}{\partial y}, \quad v=-\frac{\partial \psi}{\partial x} \quad \frac{\partial v}{\partial x}-\frac{\partial u}{\partial y}=\omega$

In order to identify the relevant dimensionless parameters influencing the problem at hand. The variable are non-dimensionalized by defining the following parameters:

$\hat{x}=\frac{x}{L} \quad \hat{y}=\frac{y}{L} \quad \hat{S}=\frac{S}{L} \quad \hat{\psi}=\frac{\psi \operatorname{Pr}}{\vartheta} \quad \Omega=\frac{\omega L^{2} \operatorname{Pr}}{\vartheta} \quad \theta=\frac{T-T_{c}}{T_{h}-T_{c}} \quad \operatorname{Ra}=\frac{\beta g\left(T_{h}-T_{c}\right) L^{3} \operatorname{Pr}}{\vartheta^{2}}$

The width of the enclosure (L) is the characteristics length in the problem.

Using non-dimensional variables defined in the above, the non-dimensional governing equations are obtained as :

Continuity Equation

$\frac{\partial^{2} \hat{\psi}}{\partial \hat{x}^{2}}+\frac{\partial^{2} \hat{\psi}}{\partial \hat{y}^{2}}=-\Omega$

Energy Equation

$\frac{\partial \hat{\psi}}{\partial \hat{x}} \frac{\partial \theta}{\partial \hat{y}}-\frac{\partial \hat{\psi}}{\partial \hat{y}} \frac{\partial \theta}{\partial \hat{x}}=\frac{\partial^{2} \theta}{\partial \hat{x}^{2}}+\frac{\partial^{2} \theta}{\partial \hat{y}^{2}}$
$\frac{\partial \hat{\psi}}{\partial \hat{x}} \frac{\partial \theta}{\partial \hat{y}}-\frac{\partial \hat{\psi}}{\partial \hat{y}} \frac{\partial \theta}{\partial \hat{x}}=\frac{\partial^{2} \theta}{\partial \hat{x}^{2}}+\frac{\partial^{2} \theta}{\partial \hat{y}^{2}}$

$\frac{\partial^{2} \theta}{\partial x^{2}}+\frac{\partial^{2} \theta}{\partial y^{2}}=0$ 
Momentum Equation

$$
\frac{\partial^{2} \hat{\psi}}{\partial \hat{x}^{2}}+\frac{\partial^{2} \hat{\psi}}{\partial \hat{y}^{2}}=R a^{*} \frac{\partial \theta}{\partial \hat{x}}
$$

$$
\frac{1}{\operatorname{Pr}}\left(\frac{\partial \hat{\psi}}{\partial \hat{x}} \frac{\partial \Omega}{\partial \hat{y}}-\frac{\partial \hat{\psi}}{\partial \hat{y}} \frac{\partial \Omega}{\partial \hat{x}}\right)=R a \frac{\partial \theta}{\partial \hat{x}}+\frac{\partial^{2} \Omega}{\partial \hat{x}^{2}}+\frac{\partial^{2} \Omega}{\partial \hat{y}^{2}}
$$

Where

$R a^{*}=R a \times D a$

$D a=\frac{K}{L^{2}}$

Boundary conditions:

$$
\begin{array}{llccc}
\text { at } & \hat{x}=0.0 & 0<\hat{y}<1 & \frac{\partial \theta}{\partial \hat{x}}=0.0 & \hat{\psi}=0.0 \\
\text { at } & \hat{x}=1.0 & 0<\hat{y}<1 & \frac{\partial \theta}{\partial \hat{x}}=0.0 & \hat{\psi}=0.0 \\
\text { at } & \hat{y}=0.0 & \hat{x}=S & \theta=1.0 & \hat{\psi}=0.0 \\
\text { at } & \hat{y}=0 & S<\hat{x}<1 & \frac{\partial \theta}{\partial \hat{y}}=0.0 & \hat{\psi}=0.0 \\
\text { at } & \hat{y}=1.0 & 0<\hat{x}<1 & \theta=0.0 & \hat{\psi}=0.0
\end{array}
$$

\section{Numerical solution:}

The governing equations for steady state, laminar, two dimensional natural convection heat transfer in an enclosure are simplified and changed to non dimensional term and then solved using finite difference method based on Taylor series. In this method, all partial derivatives in governing equations are converted to forms that can be handled by computer.[13]

The heat transfer from the walls of the enclosure can be obtained using the integration of temperature gradient along the wall and then calculate the Nusselt number as following:

$$
N u=\int_{0}^{1}\left(\frac{\partial \theta}{\partial y}\right)_{y=0,1} d x
$$

\section{Results and discussion:}

Numerical analysis of natural convection heat transfer and fluid flow was performed to obtain effects of the fluids on each other and the effect of heat source length which is located on the bottom wall of the enclosure, the upper surface of the enclosure is kept at lower temperature while remaining walls are adiabatic. Heat transfer and flow structure 
represented by stream function and isotherms line, and the effects of the parameters on heat transfer represented by Nusselt numbers.

Figure (2) shows the effect of the Rayleigh number ( $\mathrm{Ra}^{*}$ and $\mathrm{Ra}$ ) on the structure of flow and isotherm lines, from figure can notice that the increasing in $\left(\mathrm{Ra}^{*}\right)$ causes increasing in the stream function in both sides of the enclosure air and porous media sides also the temperature gradient increase along the hot and cold surface this return to increasing in the driving force ( buoyancy forces ), in the porous media side the flow become multi-cellular due to stronger circulation resulting from the increasing in the Rayleigh number.

Figure (3) this figure illustrate the effect of the dimensionless heated surface length on the structure of flow and isotherm lines from figure it could be concluded that the increasing in dimensionless heat surface length causes increasing in stream function and isotherm lines and the flow become multi-cellular due to stronger circulation because the increasing in the hot surface area causes an increasing in driving force ( buoyancy forces ).

Figure (4) shows the variation of the Nusselt number with the Rayleigh number ( $\left.\mathrm{Ra}^{*}\right)$ in porous medium side of the enclosure for different value of Rayleigh number in air side of the enclosure. The figure shows that the increasing in the Rayleigh number $\left(\mathrm{Ra}^{*}\right)$ in porous media side causes an increasing in the Nusselt number. Also the increasing in the Rayleigh number $(\mathrm{Ra})$ in the air side causes an increasing in the Nusselt number. The increasing in the Rayleigh number in the both cases resulting in increasing temperature gradient along the hot and cold surface, subsequently the heat transfer by natural convection increases and then the Nusselt number increase.

Figure (5) describes the variation of the of Nusselt number with Rayleigh number ( $\left.\mathrm{Ra}^{*}\right)$ for different value of the dimensionless heat source length $(0.2,0.4,0.5$ and 0.6$)$ and it can noted that the increasing in dimensionless heat surface length causes increasing in Nusselt number for the same Rayleigh number ( $\mathrm{Ra}^{*} \& \mathrm{Ra}$ ) in air and porous medium sides, because the natural enhanced with the increasing in dimensionless heat source length.

\section{Conclusions}

The finite difference approximation method is used to solve the governing equations ( continuity, momentum and energy ) for natural convection and fluid flwo inside two dimensional enclosure partially heated from below. the results show

1-The increasing in Rayleigh number ( $\left.\mathrm{Ra}^{*}\right)$ causes increasing in value of stream function, temperature gradient and Nusselt number and the flow become multi-cellular in porous media side.

2-The increasing in Rayleigh number $(\mathrm{Ra})$ causes increasing in value of stream function, temperature gradient and Nusselt number.

3-The increasing in dimensionless length of the heat source causes increase in stream function, temperature gradient and Nusselt number. 


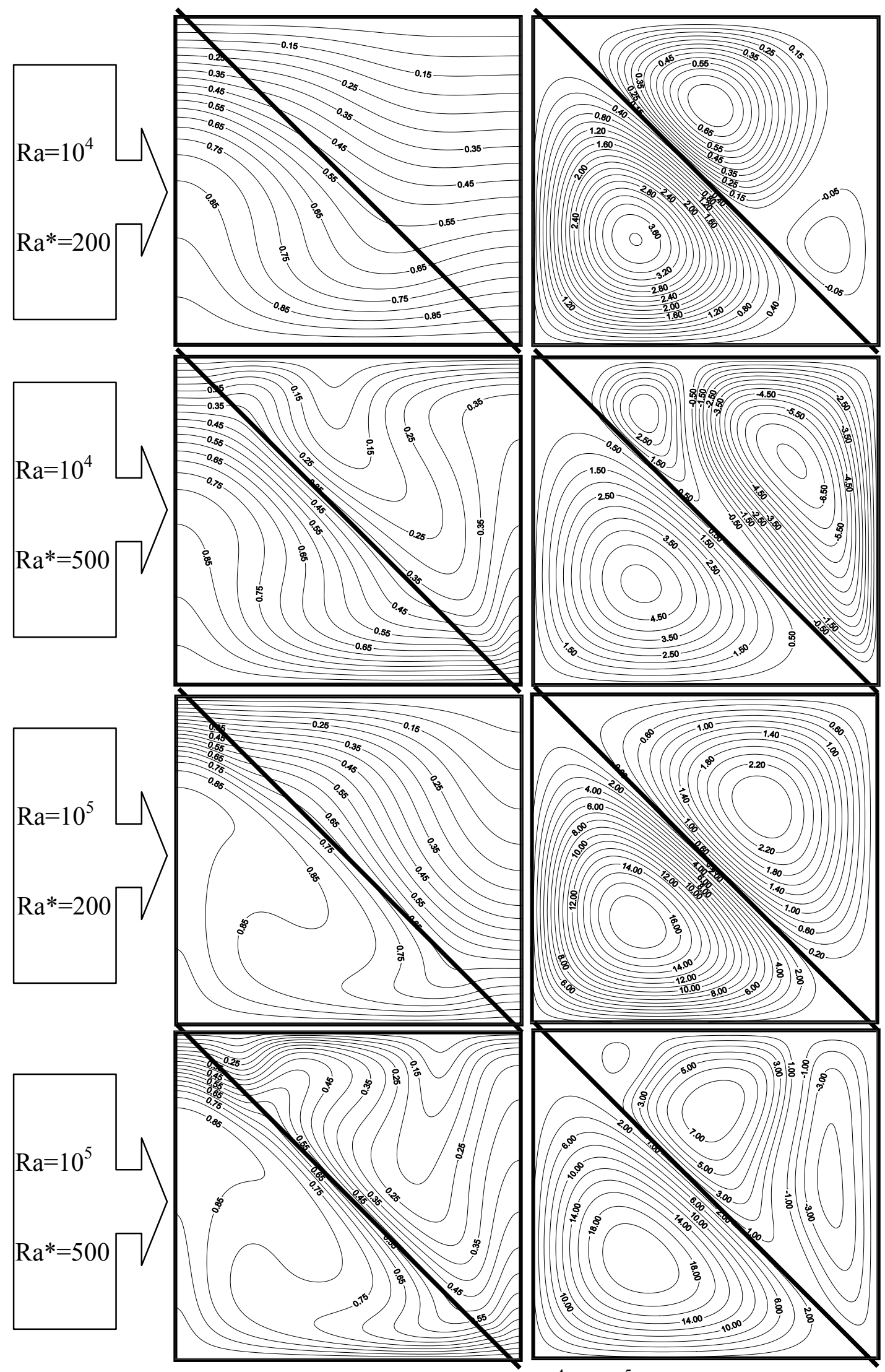

Figure (2) show the effect of $\left(\mathrm{Ra}=10^{4} \& 10^{5}\right)\left(\mathrm{Ra}^{*}=200,500\right)$ on the isotherm line and stream function 
No. 1

FEB. 2015

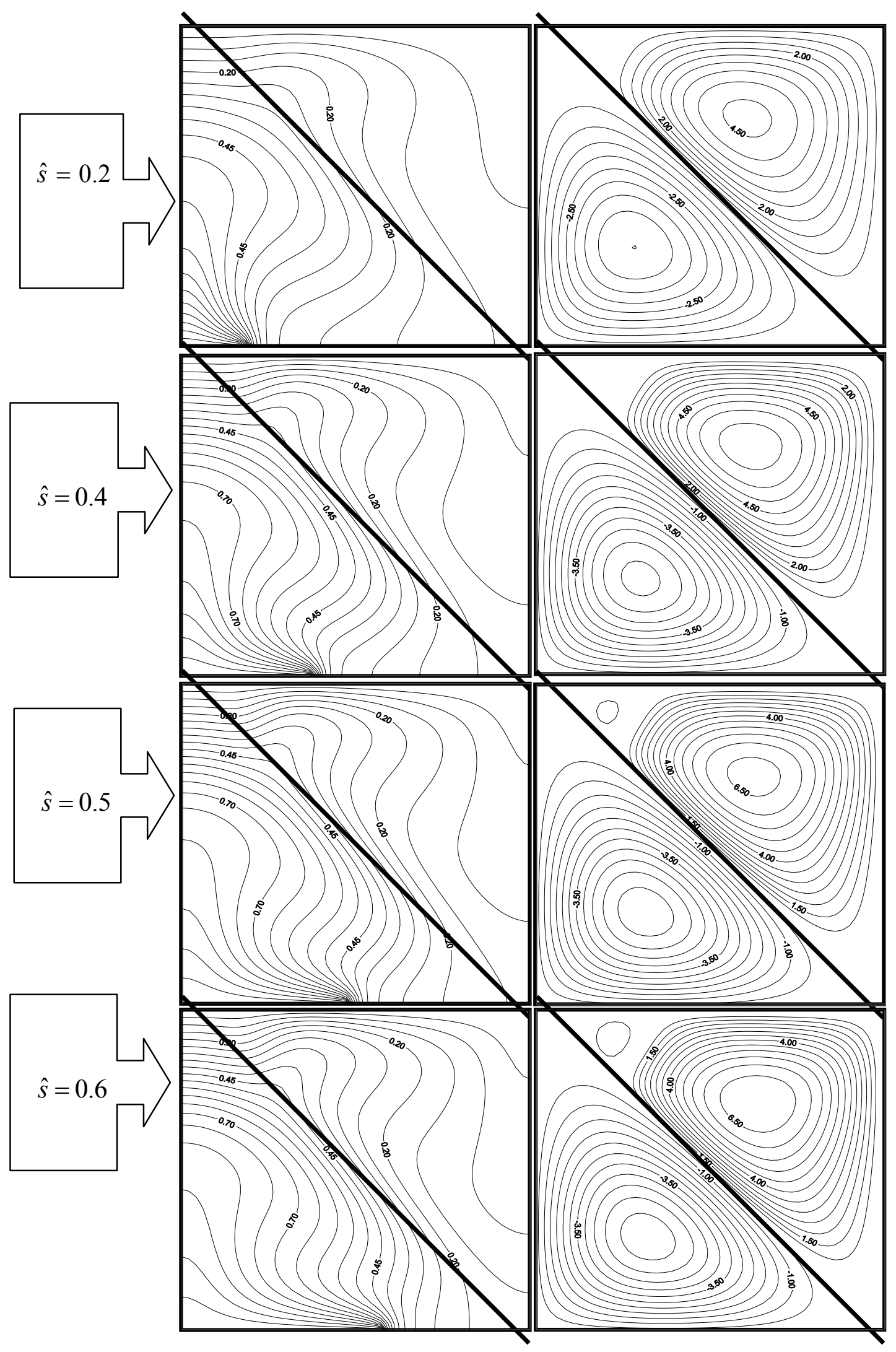

Figure (3) show the effect of dimensionless heat source length $(0.2,0.4,0.5,0.6)$ on isotherm line and stream function for $\mathrm{Ra}=10^{4}$ and $\mathrm{Ra}^{*}=500$ 


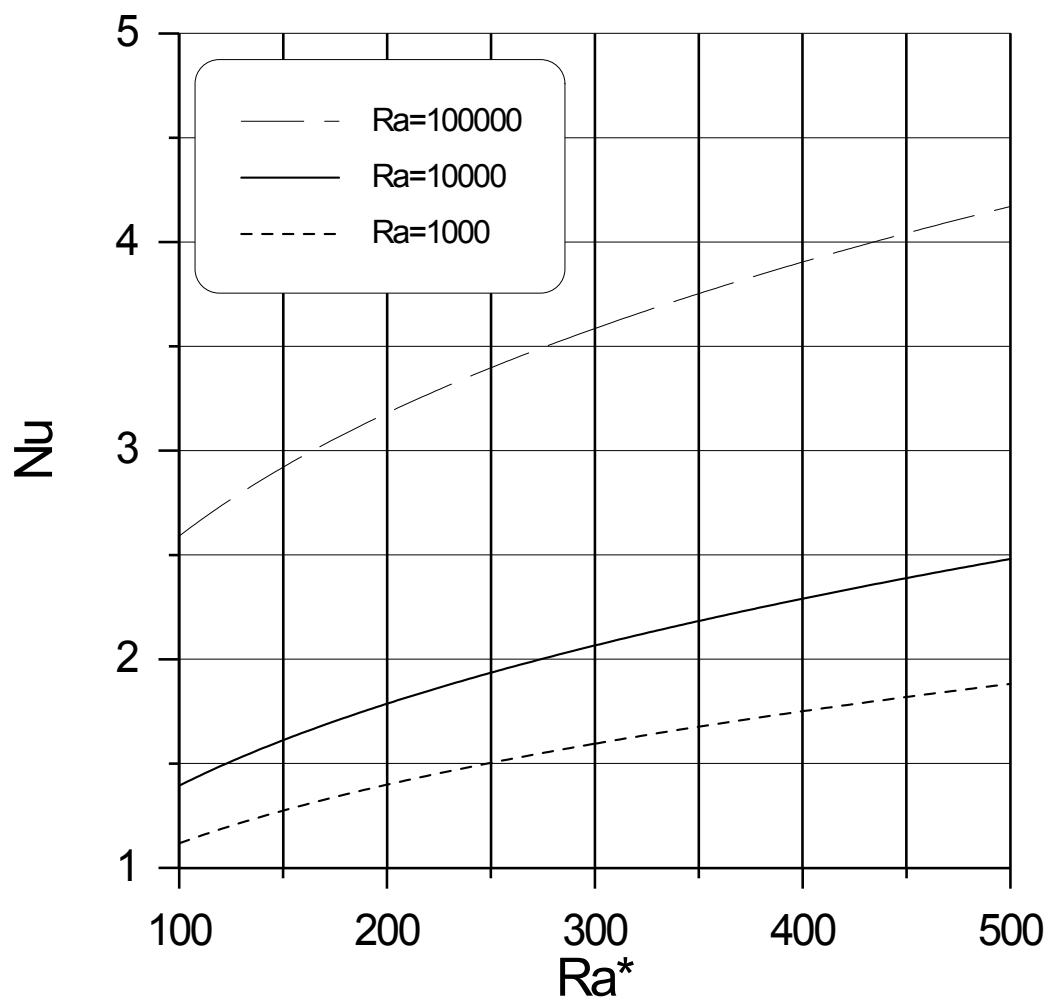

Figure ( 4 ) shows the effect of $\mathrm{Ra}^{*}$ on the $\mathrm{Nu}$ for different values of ( $\mathrm{Ra}=10^{3}, 10^{4}$ and $\left.10^{5}\right)$ and dimensionless heat source length $(\hat{s}=1)$

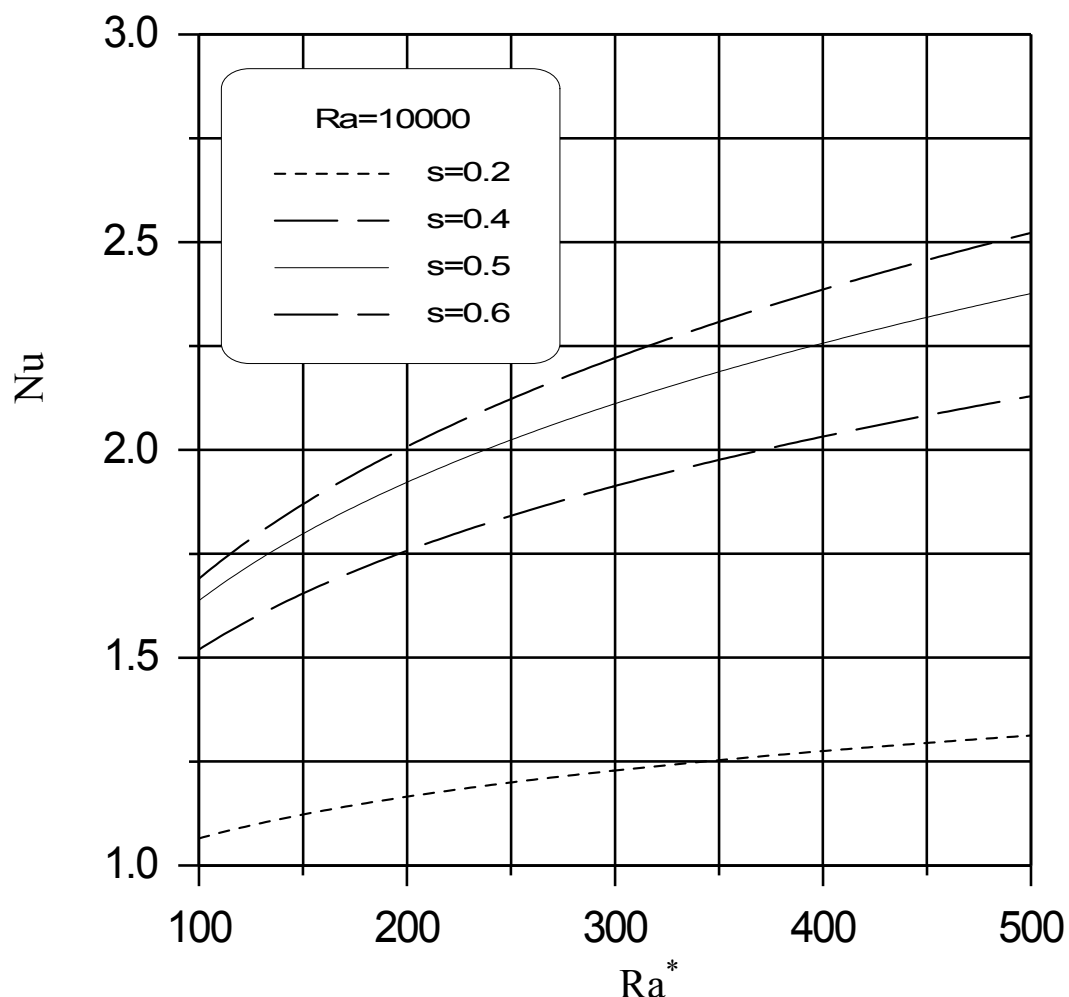

Figure ( 5 ) shows the effect of $\mathrm{Ra}^{*}$ on the $\mathrm{Nu}$ for different dimensionless heat source length $(\hat{s}=0.2,0.4,0.5$ and 0.6$)$ and $\mathrm{Ra}=10^{4}$ 


\section{References}

1- A. K. Singh , G. R. Thorpe "Natural convection in confined fluid overlying a porous layer - a comparison study of different model" Indian J. pure appl. Math., 26(1):81-95, January 1995

2-Yasin Varol " Natural convection in divided trapezoidal cavities filled with fluid saturated porous media " International Communications in Heat and Mass Transfer 37 (2010) 13501358

3-A.M. Aramayo, S. Esteban and L. Cardon "Conjugate Heat Transfer in a two Stage Trapezoidal Cavity Stack" Latin American Applied Research 39:1-9 (2009)

4-Hakan F. Oztop, Yasin Varol *, Ahmet Koca " Natural convection in a vertically divided square enclosure by a solid partition into air and water regions" International Journal of Heat and Mass Transfer 52 (2009) 5909-5921

5-Hakan F. Oztop a, Zepu Zhao b, Bo Yub "Conduction-combined forced and natural convection in lid-driven enclosures divided by a vertical solid partition" International Communications in Heat and Mass Transfer 36 (2009) 661-668

6-M. Mamou, M. Hasnaoui, P. Vasseur, and E. Bilgen " Natural Convection Heat transfer in Inclined Enclosures with Multiple Conducting Solid Partition" Numerical Heat Transfer, Part A, 25: 295-315, 1994

7-C. Beckermann S. Ramadhyani R. Viskanta "Natural Convection Flow and Heat Transfer Between a Fluid Layer and a Porous Layer Inside a Rectangular Enclosure " Journal of Heat Transfer MAY 1987, Vol. 109/363

8-Nikolay P. Moshkin" Numerical model to study natural convection in a rectangular enclosure filled with two immiscible fluids " International Journal of Heat and Fluid Flow 23 (2002) 373-379

9-Aniruddha Bagchi , F.A. Kulacki " Natural convection in fluid-superposed porous layers heated locally from below " International Journal of Heat and Mass Transfer 54 (2011) 3672-3682

10-M. Jafari, B. Ghasemi, A. Raisi, S.M. Aminossadati" Natural Convection in two Porous Media Separated by A Solid Wall" International Journal of Heat and technology 28 (2010)

11-Zineddine Alloui - Patrick Vasseur "Convection in superposed fluid and porous layers" 7 March 2010 (C) Springer-Verlag 2010

12-M. Bargach and P. Vasseur " natural convection a shallow cavity containing two superimposed layers of immiscible liquids" Numerical Heat Transfer, Part A, vol. 24, pp. 357-373, 1993

13-William F. Ames "Numerical Method for Partial differential Equation" NewYork San Francisco 1977

The work was carried out at the college of Engineering. University of Mosul 